\title{
Upregulated microRNA-330-3p promotes calcification in the bicuspid aortic valve via targeting CREBBP
}

\author{
RUI ZHENG, HAO LIU, JIAXI GU, BUQING NI, HAOLIANG SUN, YAOJUN GUO, \\ CHEN SU, KESHUAI HE, JUNJIE DU and YONGFENG SHAO \\ Department of Cardiovascular Surgery, The First Affiliated Hospital of Nanjing \\ Medical University, Nanjing, Jiangsu 210029, P.R. China
}

Received November 18, 2019; Accepted May 1, 2020

DOI: $10.3892 / \mathrm{mmr} .2020 .11297$

\begin{abstract}
One key risk factor of aortic valve stenosis in clinical practice is bicuspid aortic valve (BAV). Increasing evidence indicates that numerous microRNAs (miRs/miRNAs) are involved in BAV calcification via their target genes. miR-330-3p was found to be involved in the deterioration of BAV calcification by miR profiling in human calcified BAV and tricuspid aortic valve (TAV) tissues in the present study and the underlying mechanism was investigated. RNA sequencing was performed on four BAV and four TAV tissues from patients with aortic stenosis before these leaflets were examined for the expression levels of miR-330-3p and CREB-binding protein (CREBBP) by reverse transcription-PCR. The alteration of functional factors associated with calcification was also assessed by Western blotting and immunohistochemistry in human aortic tissue samples. The putative target of miR-330-3p was detected by dual-luciferase assay in 293 cells. Furthermore, the influence of miR-330-3p expression on osteogenic progression was explored in cultured porcine valve interstitial cells (VICs). Rescue experiments of CRBBP were performed to confirm the influence of the miR-330-3p-CREBBP pathway in the calcification progress in porcine VICs. RNA sequencing indicated distinct expression of miR-330-3p in human BAV tissues compared with TAV, which was then confirmed by PCR. CREBBP expression levels in human BAV and TAV leaflets also demonstrated the opposite alterations. This negative correlation was then confirmed in cultured porcine VICs. Under an osteogenic environment, cellular calcification was promoted in miR-330-3p-overexpressed porcine VICs
\end{abstract}

Correspondence to: Dr Junjie Du or Dr Yongfeng Shao, Department of Cardiovascular Surgery, The First Affiliated Hospital of Nanjing Medical University, 300 Guangzhou Road, Nanjing, Jiangsu 210029, P.R. China

E-mail: junjie.du@njmu.edu.cn

E-mail: yongfengshao30@hotmail.com

Key words: bicuspid aortic valve, calcification, fibrosis, microRNA-330-3p, CREB-binding protein expressing higher bone morphogenetic protein 2, Runt-related transcription factor 2, matrix metalloproteinase (MMP)-2, MMP-9 and collagen I compared with controls. Rescue experiments further confirmed that miR-330-3p played its role via targeting CREBBP in porcine VICs. Collectively, miR-330-3p was upregulated in calcified BAV compared with TAV. The upregulation of miR-330-3p promotes the calcification progress partially via targeting CREBBP.

\section{Introduction}

Aortic valve stenosis (AS) involving aortic valve calcification is the most common type of acquired valve diseases worldwide $(1,2)$. A key risk factor of AS in clinical practice is bicuspid aortic valve (BAV), which occurs in $1-2 \%$ of the general population $(3,4)$. By definition, a BAV consists of two functional leaflets, while a normal tricuspid aortic valve (TAV) contains three (4). The progression of valve calcification in adults with BAV is similar to that observed in patients with TAV; however, calcification with BAV is faster and often more severe (3). Thus, it remains critical to investigate the differences between calcified BAVs and calcified TAVs to identify key features that are associated with the deterioration of valve calcification.

The progression of valve calcification is predominantly regulated by valve interstitial cells (VICs) that populate the interstitial matrix, and is accompanied by their changes in the expression of different genes (5-8), including bone morphogenetic protein 2 (BMP2), NOTCH 1, and the SMADs, which result in differences in calcification-associated protein expression and activities (9-12). microRNAs (miRs/miRNAs) are well-known regulators of gene expression at the post-transcriptional level $(13,14)$, and growing evidence suggested that several miRs are involved in valve calcification via their target genes $(13,15-17)$. The present study performed miR profiling in calcified BAV and TAV tissues, in order to compare their differentially expressed miRs. The differentially expressed miRs were further analyzed, in which miR-330-3p was demonstrated to be involved in the deterioration of calcification of BAVs. The present study aimed to investigate the potential signaling pathways associated with upregulated miR-330-3p in BAVs and its calcification-associated target gene, CREB-binding protein (CREBBP). 


\section{Materials and methods}

Study population and aortic valve collection. The present study was approved by the Ethics Committee of the First Affiliated Hospital of Nanjing Medical University, and written informed consent was obtained from all patients prior to the study. Aortic leaflets were collected from patients receiving aortic replacement between January 2016 and December 2018 at Jiangsu Province Hospital. Echocardiography was performed to confirm the morphology and function of the leaflets prior to surgery. A total of $15 \mathrm{BAV}$ samples were examined in the present study, all of which presented type $1 \mathrm{BAV}$ leaflets, with fusion of the right and left coronary leaflets. A total of 15 degenerative TAVs were selected according to the echocardiographic results and intra surgical inspections. All leaflets were sectioned into two parts. The larger part was flash frozen in liquid nitrogen and stored for RNA or protein extraction, while the smaller part was fixed in 10\% formalin and embedded in paraffin for subsequent experimentation.

miRNA microarray. Agilent Human miRNA Microarray Slide (version 21.0; 8x60K; Design ID: 070156; Agilent Technologies, Inc.) was used to profile miRNA expression in 8 aortic valve tissue samples from patients with BAV or TAV. Total RNA was quantified using a NanoDrop ND-2000 spectrophotometer (Thermo Fisher Scientific, Inc.) and the RNA integrity was assessed using an Agilent Bioanalyzer 2100 (Agilent Technologies, Inc.). The sample labeling, microarray hybridization and washing were performed based on the manufacturer's standard protocol. Briefly, total RNA was dephosphorylated, denaturated and labeled with Cyanine-3-CTP. After purification the labeled RNAs were hybridized onto the microarray. After washing, the arrays were scanned using the Agilent Scanner G2505C (Agilent Technologies, Inc.).

Feature Extraction software (version 10.7.1.1; Agilent Technologies, Inc.) was used to analyze array images to get raw data. Subsequently, Genespring software (version 13.1; Agilent Technologies, Inc.) was employed to finish the basic analysis with the raw data. To begin with, the raw data was normalized with the quantile algorithm. The probes that at least 100.0 percent of samples in any 1 condition out of 2 conditions have flags in 'Detected' were chosen for further data analysis. Differentially expressed miRNAs were then identified through fold change as well as P-value calculated using t-test. The threshold set for up- and down-regulated genes was a fold change $>=1.5$ and a P-value $<=0.05$. Target genes of differentially expressed miRNAs were the intersection predicted with 3 databases [(TargetScan (www.targetscan.org), microRNAorg (www.microrna.org) and PITA (www.pictar.org)].

Reverse transcription-quantitative PCR (RT-qPCR). miRs or mRNAs were extracted from the tissues obtained from surgical operations or cultured VICs using the miRNeasy Mini kit (Qiagen, Inc.) according to the manufacturer's instructions, and reverse transcribed into cDNA using the Takara PrimeScript ${ }^{\mathrm{TM}}$ RT master mix (Takara Bio, Inc.) according to the manufacturer's instructions. qPCR was subsequently performed using the 7900HT Fast Real-Time PCR System (Thermo Fisher Scientific, Inc.) according to manufacturer's protocol. The primer sequences used for qPCR were purchased from
Realgene Bio-Technologies, Inc. and are presented in Table I. The following thermal cycling conditions were used for the qPCR: Initial denaturation at $95^{\circ} \mathrm{C}$ for $10 \mathrm{~min}$; then 40 cycles of $95^{\circ} \mathrm{C}$ for $15 \mathrm{sec}$ and $60^{\circ} \mathrm{C}$ for $1 \mathrm{~min}$. Relative expression levels were measured using the $2^{-\Delta \Delta \mathrm{Cq}}$ method (18) and normalized to the internal reference gene GAPDH. U6 small nuclear RNA was used as internal control to normalize miRNA expression. All experiments were performed in triplicate.

Histological examination and immunohistochemistry. The tissue samples were fixed with $10 \%$ formalin at room temperature for $24 \mathrm{~h}$, embedded in paraffin and cut into $20-\mu \mathrm{m}$ thick sections for Von Kossa and Sirius Red staining and semi-quantitative analysis. Tissues were also used for immunohistochemical staining for CREBBP, collagen I, Runt-related transcription factor 2 (Runx 2), matrix metalloproteinase (MMP)-2 and MMP-9. In brief, sections were washed in Tris-buffered saline $/ 0.1 \%$ Tween- 20 and subsequently blocked in 5\% milk at room temperature for $2 \mathrm{~h}$. Tissue sections were incubated with the primary antibodies (all 1:1,000) listed in Table II at $4^{\circ} \mathrm{C}$ overnight. Sections were washed in PBS prior to incubation with biotinylated secondary antibody (1:200; cat. no. 21537; Merck KGaA) at room temperature for $30 \mathrm{~min}$. The sections were subsequently treated with Vectastain ABC reagent (Vector Laboratories, Inc.) and 3,3'-diaminobenzidine, prior to counterstaining with hematoxylin at room temperature for $2 \mathrm{~min}$. A light optical microscope was used for image capture (magnification, $\mathrm{x} 10$ or $\mathrm{x} 200$ ) using visible light source without any filters. Image-Pro plus software (version 6.0; Media Cybernetics, Inc.) was used for signal quantification. In brief, the positive area was automatically selected according to criteria that contained a specific Hue-Saturation-Intensity (HIS) parameter adjusted to the different staining method. Subsequently, the optical density (OD) of the positive areas and the whole image were calculated automatically. The data were extracted and the OD of per unit area was calculated and presented as a semi-quantitative indicator.

VIC culture and transient transfection. All procedures involving the use of animals were approved by the Institutional Animal Care and Use Committee of Nanjing Medical University. Porcine aortic VICs were harvested from porcine hearts, which were supplied by the Laboratory Animal Center of Nanjing Medical University and isolated by collagenase digestion as previously described (19). In brief, pigs at 3-4 months old were sedated using a cocktail of ketamine hydrochloride $(20 \mathrm{mg} / \mathrm{kg}$, intramuscular injection) and xylazine $(2 \mathrm{mg} / \mathrm{kg}$, intramuscular injection). Following adequate sedation ( $15 \mathrm{~min})$, pigs were euthanized by overdose of pentobarbital $(156 \mathrm{mg} / \mathrm{kg}$ body weight) administered intravenously and their hearts were harvested immediately. Aortic valves were excised with precision to exclude non-leaflet tissue. The excised leaflets were carefully scraped on the aortic and ventricular aspects to remove the endothelial layer. Tissues samples were subsequently sectioned into $\sim 2 \times 2 \mathrm{~mm}$ explants, which were cultured in DMEM (Gibco; Thermo Fisher Scientific, Inc.) supplemented with $1 \%$ penicillin-streptomycin (Beyotime Institute of Biotechnology), and 15\% fetal bovine serum (ScienCell Research Laboratories, Inc.), in $5 \% \mathrm{CO}_{2}$ at $37^{\circ} \mathrm{C}$. Cells that reached $\sim 100 \%$ confluence were extracted 
from the explants for subculture and those of the 2 nd or $3 \mathrm{rd}$ generations were used for further analyses.

An antisense-based strategy was adapted to block miRNA function in cultured cells. Anti-miR-330 2'-O-methyl oligoribonucleotides (Guangzhou RiboBio Co., Ltd.) were synthesized to sequence-specifically inactivate miR-330 and thus to release the suppression on target mRNAs without changing its expression level (20). A total of $7.5 \times 10^{4}$ cells $/ \mathrm{cm}^{2}$ VICs were transfected with $5 \mathrm{nmol} / 1 \mathrm{miR}-330-3 \mathrm{p}$ mimic or inhibitor (micrONTM miR mimic/inhibitor/nc_Standard; cat. nos. miR10000751-1-5 and miR20000751-1-5; Guangzhou RiboBio Co., Ltd.) using Lipofectamine ${ }^{\mathrm{TM}} 3000$ reagent (Invitrogen; Thermo Fisher Scientific, Inc.). The transfected cells were incubated in DMEM supplemented with 15\% FBS for $6 \mathrm{~h}$ and subsequently were incubated in DMEM supplemented with $1 \%$ penicillin-streptomycin and $15 \%$ FBS. At $48 \mathrm{~h}$ post-transfection, cells were used for subsequent experiments.

VICs were also transfected with miR-659 mimic, miR-663 mimic, or miR-146 inhibitor (Guangzhou RiboBio Co., Ltd.) using the aforementioned protocol.

Pro-osteogenic medium preparation and Alizarin Red $S$ staining for calcium deposits. To prepare the calcification medium, $1 \mathrm{mmol}$ of $\mathrm{CaCl}_{2}$ and $1 \mathrm{mmol}$ of $\mathrm{Na}_{5} \mathrm{P}_{3} \mathrm{O}_{10}$ were dissolved in $50 \mathrm{ml}$ of water to produce reagent one and reagent two, respectively. Subsequently, $500 \mu \mathrm{l}$ reagent one, $375 \mu \mathrm{l}$ reagent two and $4 \mathrm{ml}$ of $8 \%$ FBS were added to $45 \mathrm{ml}$ DMEM. VICs were cultured with or without pro-osteogenic medium for $48 \mathrm{~h}$ post-transfection, and subsequently stained with Alizarin Red S Staining (Beyotime Institute of Biotechnology) according to the manufacturer's protocol. Briefly, cells were washed with $\mathrm{Ca}^{2+}$-free PBS three times, fixed with $4 \%$ paraformaldehyde for $10 \mathrm{~min}$ at room temperature and then fixed with 95\% ethanol for $20 \mathrm{~min}$ at room temperature. Subsequently, cells were stained with $1 \%$ alizarin red solution $(\mathrm{pH} \mathrm{4.2;}$ cat. no. ST1078; Beyotime Institute of Biotechnology) for $1 \mathrm{~min}$ at room temperature to visualize matrix calcium deposition. The solution was washed out with distilled water and the stained cells were photographed using a digital camera. The images were then processed and analyzed using Image-Pro Plus software (version 6.0, Media Cybernetics, Inc.).

Western blotting. Transfected cells were harvested and used for preparation of protein extracts. Cells were lysed using a cell lysis kit (Nanjing KeyGen Biotech Co., Ltd.). The bicinchoninic acid method was used for quantification of total protein of the samples. Equal amounts of protein extracts $(30 \mu \mathrm{g})$ were separated by $10 \%$ SDS-PAGE and transferred to PVDF membranes. Following blocking with TBS-Tween-20 solution containing 5\% BSA (cat. no. B600036; Sangon Biotech Co., Ltd.) for $2 \mathrm{~h}$ at room temperature, the membranes were incubated with the following primary antibodies: Rabbit polyclonal antibody against Runx 2 (1:1,000; cat. no. 12556; Cell Signaling Technology, Inc.), rabbit polyclonal antibody against BMP2 (1:1,000; cat. no. 14933; Abcam), rabbit polyclonal antibody against CREBBP (1:1,000; cat. no. 2832; Abcam), rabbit polyclonal antibody against collagen I (1:1,000; cat. no. WL0088; Wanleibio Co., Ltd.), and mouse monoclonal antibody against GAPDH (1:1,000; cat. no. 8245; Abcam) overnight at $4^{\circ} \mathrm{C}$. The membranes were then probed using either a goat anti-rabbit or a
Table I. Primer sequences used for reverse transcription-quantitative PCR.

\begin{tabular}{ll} 
Name & \multicolumn{1}{c}{ Primer sequence (5'-3') } \\
\hline hsa-CREBBP & F: CAACCCCAAAAGAGCCAAACT \\
& R: CCTCGTAGAAGCTCCGACAGT \\
ssc-GAPDH & F: TCGGAGTGAACGGATTTGGC \\
& R: TGACAAGCTTCCCGTTCTCC \\
hsa-GAPDH & F: AGAAGGCTGGGGCTCATTTG \\
& R: AGGGGCCATCCACAGTCTTC \\
ssc-collagen I & F: GAGGGCCAAGACGAAGACATC \\
& R: CAGATCACGTCATCGCACAAC \\
hsa-collagen I & F: AGCCCTGGTGAAAATGGAGC \\
ssc-Runx 2 & R: CACCCTTAGCACCAACAGCA \\
& F: AGCCACCTACCACAGAGCTA \\
ssc-BMP2 & R: GGATGAGGAATGCGCCCTAA \\
& F: GAGCTAGCACTGAGCGACC \\
hsa-U6 & R: GAAGTCTCCAGCCAAGTGCT \\
& F: GTGGGGAGAAGAGGACAGGA \\
& R: GTGGTACCCACTTTCGCACA
\end{tabular}

CREBBP, CREB-binding protein; Runx 2, Runt-related transcription factor 2; BMP2, bone morphogenetic protein 2; F, forward; R, reverse.

goat anti-mouse horseradish peroxidase-conjugated secondary antibody (1:5,000; cat. nos. ab150077 and ab205719; Abcam) for $2 \mathrm{~h}$ at room temperature. The blots were developed with an Omni-ECL ${ }^{\mathrm{TM}}$ Enhanced Pico Light Chemiluminescence kit (Epizyme Co.) and exposed on a ChemiDoc MP imaging system (Bio-Rad Laboratories, Inc.). Band density was quantified using ImageJ software (version 1.8.0; National Institutes of Health) and normalized to GAPDH.

Dual-luciferase reporter assay. Specific culture medium was prepared by mixing DMEM, 10\% FBS (ScienCell Research Laboratories, Inc.), $1 \%$ nonessential amino acids, $1 \%$ L-glutamine and $1 \%$ penicillin with streptomycin. 293T cells (National Infrastructure of Cell Line Resource) were seeded into 12 -well plates $\left(5 \times 10^{4}\right.$ cells $\left./ \mathrm{cm}^{2}\right)$ and cultured in specific medium at $37^{\circ} \mathrm{C}$ in $5 \% \mathrm{CO}_{2}$.

The pmirGLO dual luciferase miR target expression vector (pmirGLO), containing both firefly and Renilla luciferase genes was purchased from Promega Corporation. Human CREBBP 3'-untraslated region (3'-UTR), including the predicted binding site of miR-330-3p, was amplified via RT-PCR and inserted into the 3'-UTR downstream of the firefly luciferase gene in the pmirGLO vector (pmirGLO-UTR) using $X b a \mathrm{I}$ and $S a c \mathrm{I}$ restriction sites. The QuikChange XL Site-Directed Mutagenesis kit (Agilent Technologies, Inc.) was used to construct the mutant miR-330-3p-binding site vector (pmirGLO-UTR-MUT) according to the manufacturer's protocol. Restriction enzyme digestion and sequencing was performed to validate the constructs. After cellular transfection for $36 \mathrm{~h}$, luciferase activity was assessed with Lipofectamine $^{\mathrm{TM}} 3000$ reagent (Invitrogen; Thermo Fisher Scientific, Inc.) using a Dual-Glo luciferase assay system 
Table II. Antibody information.

\begin{tabular}{llll}
\hline Name of antibody & Catalog no. & \multicolumn{1}{c}{ Supplier } & Type \\
\hline Collagen I & WL0088 & Wanleibio Co., Ltd. & Polyclonal \\
BMP2 & ab14933 & Abcam & Polyclonal \\
Runx 2 & 12556 & Cell Signaling Technology, Inc. & Monoclonal \\
GAPDH & ab8245 & Abcam & Monoclonal \\
CREBBP & ab2832 & Abcam & Polyclonal \\
MMP2 & sc-13594 & Santa Cruz Biotechnology, Inc. & Monoclonal \\
MMP9 & sc-21736 & Santa Cruz Biotechnology, Inc. & Monoclonal
\end{tabular}

BMP2, bone morphogenetic protein 2; Runx 2, Runt-related transcription factor 2; CREBBP. CREB-binding protein; MMP, matrix metalloproteinase.

(Promega Corporation). Firefly luciferase activity was normalized to Renilla luciferase activity.

Engineered clustered regularly interspaced shot palindromic repeats (CRISPR)/CRISPR associated protein 9 (-Cas9) and DNA constructs. The design and use of engineered Cas9 complex (pLenti-CMV-NLS-dCas9-VP64-2A -Puro; cat. no. H7281; Heyuan Kangning Medical Company Biotechnology Limited Company) and efficient single guide RNA (gRNA; plenti-U6-gRNA-2x(wt+f6) MS2-CMV-MCP-P65-HSF1-IRES-blasticidin; cat. no. H7284; Heyuan Kangning Medical Company Biotechnology Limited Company) to induce CREBBP transcriptional activation was performed according to previously published protocols $(21,22)$. CCTop, a CRISPR/Cas9 target online predictor (cctop.cos. uni-heidelberg.de) was used to design the gRNAs used in the present study. The sequences of both control and CREBBP gRNAs are as follows: 5'-GCACTACCAGAGCTAACTCA-3' (control gRNA) and 5'-CCACTTAATGAATTCGCTCG-3' (CREBBP gRNA). The gRNA primers were annealed and cloned into gRNA (MS2)-plasmids via the BbsI sites. All CRISPR constructs were purchased from Heyuan Biotech Company (cat. nos. 61422, 61423 and 61424). The CREBBP promoter-luciferase construct was generated by synthesizing the DNA fragment corresponding to the CREBBP promoter region from GenScript, and sub-cloned into the pGL4.20 vector (Heyuan Biotech Company).

Statistical analysis. Statistical analysis was performed using SPSS software (version 19.0; IBM Corp.). Univariate analyses were performed using the $\chi^{2}$ test for categorical variables, and Student's t-test or one-way ANOVA followed by Dunnett's post hoc were performed for continuous variables. Pearson's correlation analysis was used to investigate the relationship between two quantitative, continuous variables. Data are presented as the mean $\pm \mathrm{SD}$. $\mathrm{P}<0.05$ was considered to indicate a statistically significant difference.

\section{Results}

Clinical characteristics of patients with BAV and TAV. Clinical characteristics of the patients involved in the present study are listed in Table III. Patients with BAV were significantly younger compared with patients with TAV. No significant differences were observed between the two groups in sex and other risk factors concerning cardiovascular calcification. Both groups presented similar preoperative echocardiography results, which measured the left ventricular ejection fraction, aortic valve area and mean trans valvular gradient. Based on the echocardiography results, patients with a mean aortic valve area $<0.75 \mathrm{~cm}^{2}$ were diagnosed with severe AS.

Differences in miR expression profiles of valve tissue from $A S$ patients with $B A V$ and $T A V$. Valve tissues from patients with AS, with either BAV $(n=4)$ or TAV $(n=4)$ were used for miR profiling. miRs that were commonly upregulated or downregulated in patients with BAV compared with patients with TAV were selected, including 11 upregulated and eight downregulated genes, respectively (Table IV). The heat map of the differentially expressed miRs are presented in Fig. 1A.

miR sequencing results of the patients' valve tissues were validated via RT-qPCR analysis. miR-330-3p, miR-659 and miR-663 expression levels in the BAV leaflets were significantly increased compared with the degenerative TAV leaflets by 3.72-fold, 5.04-fold and 4.22-fold, respectively (Fig. 1B-D); however, miR-146 expression was significantly decreased by -11.56-fold (Fig. 1E). No significant differences were observed in the other 15 miRs (Fig. S1).

Calcification progress is accelerated by miR-330-3p in sus scrofa VICs. VICs were successfully transfected with miR-659 or miR-663 mimics or miR-146 inhibitor as the expression of these miRNAs were significantly increased (miR-659 and miR-663) or decreased (miR-146) following transfection (Fig. S2A, E and I). However, levels of calcification-associated factors Runx 2 and BMP2 and the fibrosis-associated factor collagen I in miR-659 mimic- and miR-663 mimic-transfected cells did not show any significant difference compared with controls (Fig. S2B-D, F-H). In addition, mRNA expression levels of Runx 2 and BMP2 were significantly decreased in miR-146 inhibitor-transfected cells compared with controls (Fig. S2J and K). As the simulation of tissue expression alterations to the three microRNAs in cultured VICs failed to demonstrate similar calcification-associated protein alterations to those observed in BAV tissue samples, further experimentation on miR-659, miR-663 and miR-146 was ceased. 
Table III. Patient demographics.

\begin{tabular}{|c|c|c|c|}
\hline Variable & TAV $(n=15)$ & BAV $(n=15)$ & P-value \\
\hline Age (years) & $61.40 \pm 8.91$ & $49.20 \pm 10.72$ & 0.00 \\
\hline Females, \% (n) & $46.67(7)$ & $53.33(8)$ & 0.72 \\
\hline \multicolumn{4}{|l|}{ Risk factors } \\
\hline Hypertension, \% (n) & $37.50(3)$ & $25.00(2)$ & 1.00 \\
\hline Hypercholesterolemia, \% (n) & $50.00(4)$ & $62.50(5)$ & 1.00 \\
\hline Diabetes, \% (n) & $37.50(3)$ & $50.00(4)$ & 1.00 \\
\hline Smoking history, \% (n) & $25.00(2)$ & $37.50(3)$ & 1.00 \\
\hline Chronic kidney disease, \% (n) & $0.00(0)$ & $0.00(0)$ & $\mathrm{N} / \mathrm{A}$ \\
\hline \multicolumn{4}{|l|}{ Echocardiographic parameters } \\
\hline Left ventricular ejection fraction, $\%$ & $63.25 \pm 4.61$ & $62.99 \pm 5.71$ & 0.89 \\
\hline Flow velocity maximum of aortic valve, $\mathrm{m} / \mathrm{s}$ & $4.56 \pm 0.88$ & $4.20 \pm 0.86$ & 0.48 \\
\hline Mean gradient, $\mathrm{mmHg}$ & $70.27 \pm 26.33$ & $65.33 \pm 34.21$ & 0.70 \\
\hline Ascending aorta maximal diameter, $\mathrm{mm}$ & $40.23 \pm 4.42$ & $44.83 \pm 5.92$ & 0.07 \\
\hline
\end{tabular}

BAV, bicuspid aortic valve; TAV, tricuspid aortic valve.

Table IV. Differentially expressed microRNAs of aortic valves from patients with BAV compared with patients with TAV.

A, Downregulated differentially expressed genes

\begin{tabular}{lcr}
\hline & $\begin{array}{c}\text { P-value } \\
\text { microRNA }\end{array}$ & Fold change \\
\hline hsa-miR-1228-5p & 0.038 & 3.195 \\
hsa-miR-146a-5p & 0.036 & 3.104 \\
hsa-miR-3149 & 0.027 & 15.946 \\
hsa-miR-32-3p & 0.026 & 13.893 \\
hsa-miR-4455 & 0.022 & 1.676 \\
hsa-miR-5001-5p & 0.031 & 1.645 \\
hsa-miR-574-5p & 0.045 & 1.763 \\
hsa-miR-6068 & 0.044 & 1.537 \\
hsa-miR-6125 & 0.015 & 1.597 \\
hsa-miR-663a & 0.020 & 1.518 \\
hsa-miR-6847-5p & 0.035 & 1.575 \\
\hline
\end{tabular}

B, Upregulated differentially expressed genes

\begin{tabular}{lcc}
\hline & $\begin{array}{c}\text { P-value } \\
\text { microRNA }\end{array}$ & Fold-change \\
\hline hsa-miR-215-5p & 0.036 & 6.212 \\
hsa-miR-3158-5p & 0.017 & 11.207 \\
hsa-miR-330-3p & 0.041 & 4.462 \\
hsa-miR-3610 & 0.026 & 21.066 \\
hsa-miR-451a & 0.038 & 6.534 \\
hsa-miR-6081 & 0.017 & 9.466 \\
hsa-miR-659-3p & 0.045 & 12.583 \\
hsa-miR-887-3p & 0.030 & 12.512 \\
\hline
\end{tabular}

miR, microRNA; BAV, bicuspid aortic valve; TAV, tricuspid aortic valve.
To investigate whether miR-330-3p plays a role in cellular calcification, VICs transfected with miR-330 mimic or inhibitor were incubated under pro-osteogenic conditions (Fig. 2A). After 48 h, both under pro-osteogenic conditions, a significantly higher degree of calcification was observed in the miR-330-3p mimic group compared with the miR-330-3p negative control group, while cells transfected with miR-330 inhibitor demonstrated a significant decrease of calcification compared with the miR-330-3p negative control group (Fig. 2A). Higher collagen I mRNA expression was observed in VICs treated with miR-330-3p mimic compared with the control group (Fig. 2B), indicating that the fibrosis progress was accelerated by miR-330, thus fibrosis is considered a pro-calcification progress. Concurrently, calcification-associated pathways were investigated at the mRNA level. VICs exhibited a 2.88-fold increase in BMP2 mRNA expression in the miR-330 mimic group compared with the control group (Fig. 2B). Similar alteration patterns were demonstrated in the expression of the downstream pro-osteogenic transcription factor, Runx 2 (7.95-fold increase vs. control; Fig. 2B). Alternatively, VICs transfected with miR-330 inhibitor demonstrated a significant decrease in mRNA expression of collagen I, BMP2 and Runx 2 compared with their respective control groups (Fig. 2C).

Furthermore, the protein expression of collagen I, BMP2 and Runx 2 significantly increased in cells transfected with miR-330-3p mimic compared with the control groups following pro-calcification culture, indicating that the calcification progress was accelerated in these cells (Fig. 3A and B). VICs transfected with miR-330-3p inhibitor showed decreased protein expression levels of BMP2 and Runx 2 compared with the control group; however, protein expression level of collagen 1 increased (Fig. 3A and C). Western blots from different experiments are shown in Fig. S3A and B. Taken together, these results suggested that miR-330-3p might facilitate the calcification progress in cultured VICs. 


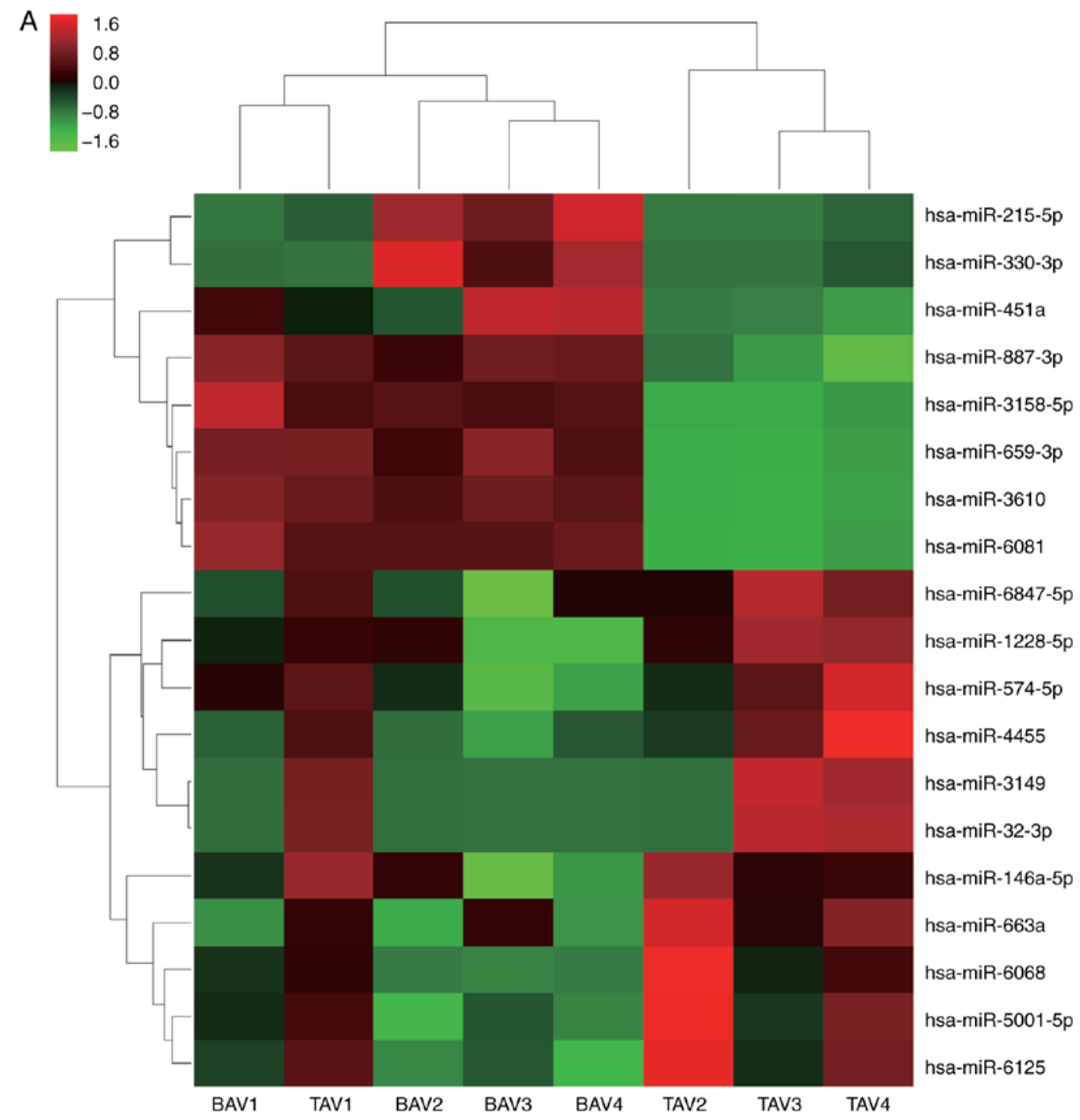

B

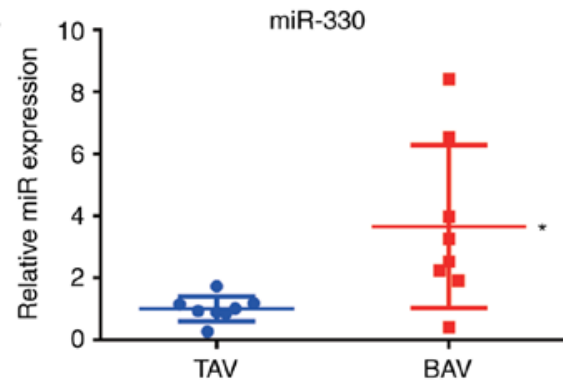

D

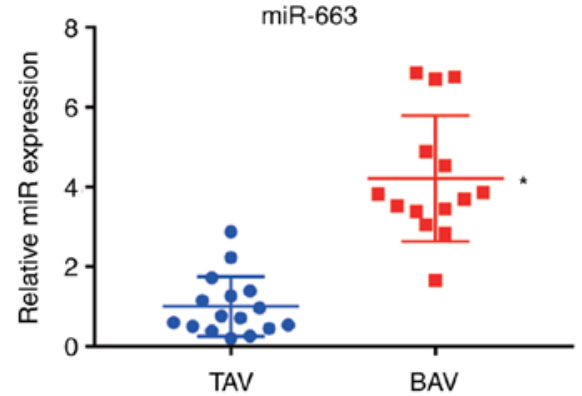

C

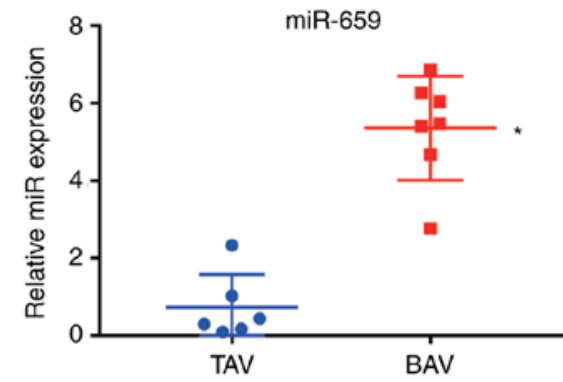

E

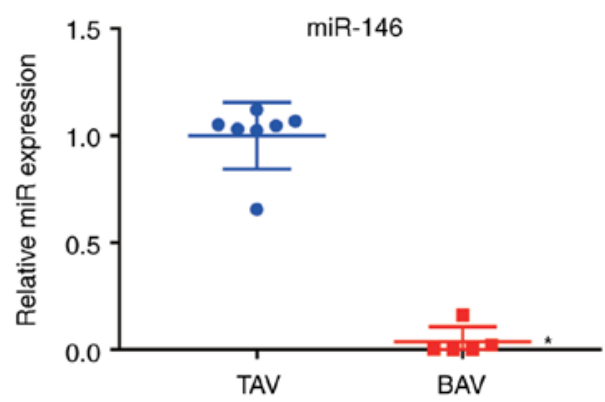

Figure 1. Differentially expressed miRNAs in patients with BAV and TAV. (A) Heat map conveying the significantly differentially expressed miRNAs between the human BAV and TAV tissue samples ( $n=4$ per group). Columns represent samples and rows represent miRNAs. Red represents upregulated genes, green represents downregulated genes and black represents no change in gene expression. The sample ID is indicated at the bottom of the heat map. Samples were arranged by the clustering of gene expression patterns. Reverse transcription-quantitative PCR analysis results on the expression of (B) miR-330-3p, (C) miR-659, (D) miR-663 and (E) miR-146 in aortic valve tissues from patients with BAV and TAV (n=15 per group). Student's t-test was performed. Data are presented as the mean \pm standard deviation. ${ }^{*} \mathrm{P}<0.05$ vs. TAV. miR, microRNA; BAV, bicuspid aortic valve; TAV, tricuspid aortic valve. 
A

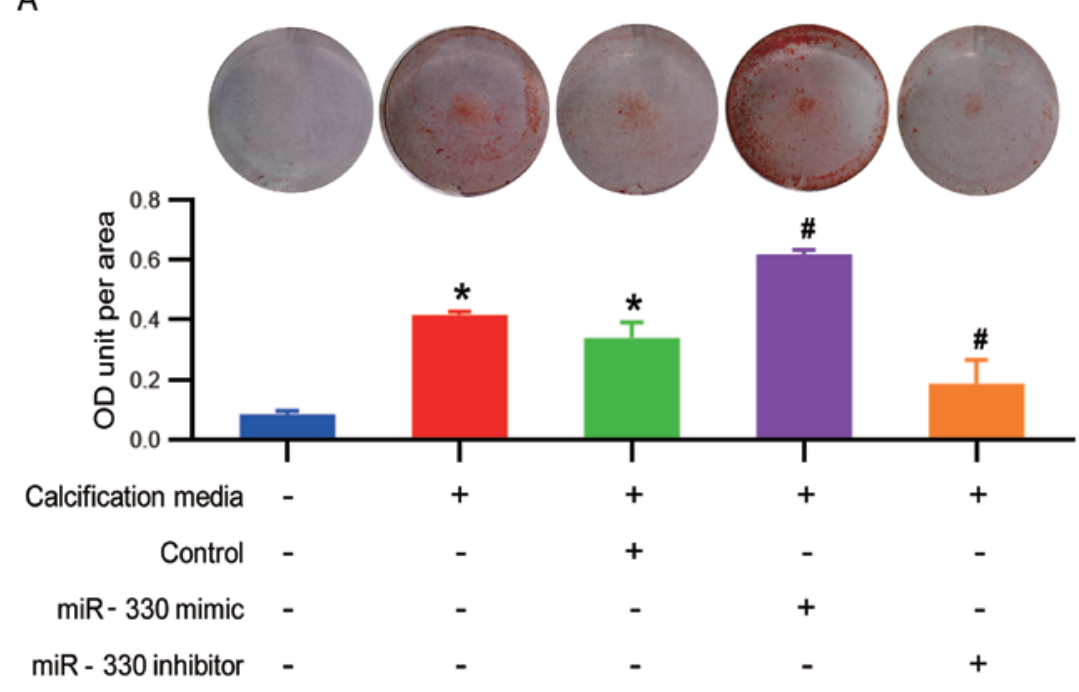

B
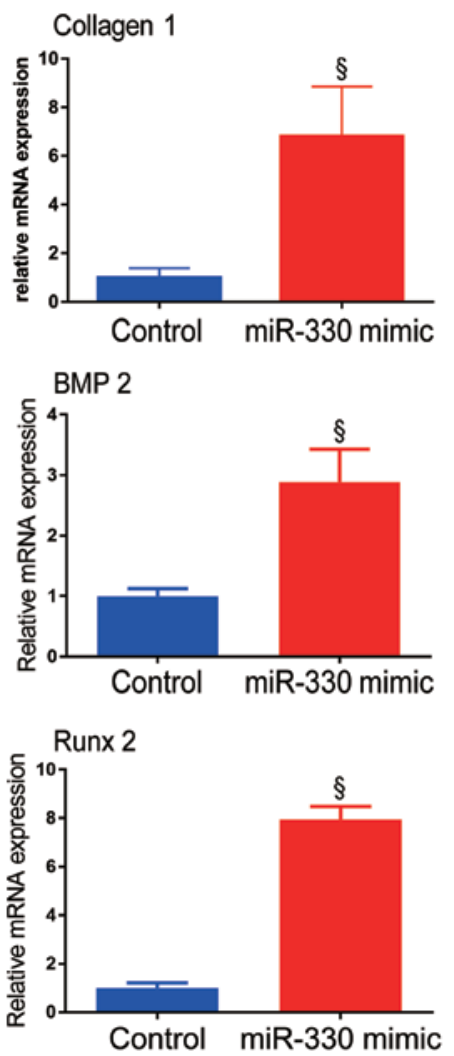

C
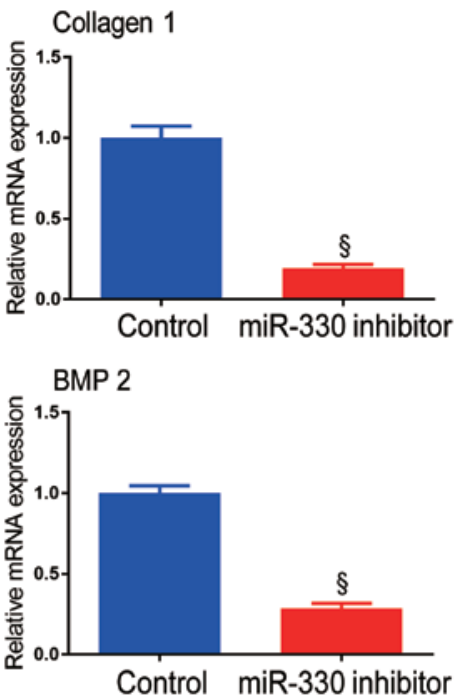

Runx 2

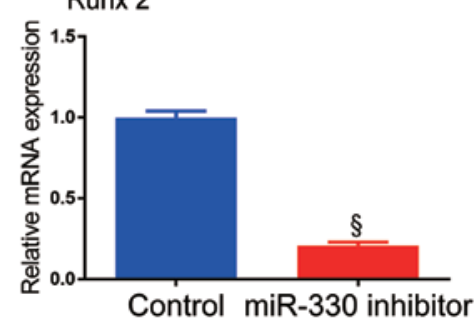

Figure 2. miR-330 facilitates the calcification progress in cultured VICs. (A) Representative images and quantification of Alizarin Red S staining for calcium deposition in cultured VICs transfected with miR-330 mimic or miR-330 inhibitor. One-way ANOVA with Dunnett's post hoc test was performed. ${ }^{*} \mathrm{P}<0.05$ vs. negative control without calcification media; ${ }^{~} \mathrm{P}<0.05$ vs. negative control with calcification media. mRNA expression of collagen $1, \mathrm{BMP} 2$, and Runx 2 in VICs transfected with (B) miR-330 mimic or (C) miR-330 inhibitor. Student's t-test was performed. ${ }^{\S} \mathrm{P}<0.05$ vs. control. Data are presented as the mean \pm standard deviation. miR, microRNA; BMP2, bone morphogenetic protein 2; Runx 2, Runt-related transcription factor 2; VICs, valvular interstitial cells; OD, optical density.

CREBBP is a direct target of miR-330-3p. Based on online bioinformatics analysis using TargetScan software (www. targetscan.org, version 7.0) and relevant studies investigating vascular calcification $(23,24)$, CREBBP was identified as a potential target gene of miR-330-3p. Immunohistochemical staining of CREBBP was performed in human aortic valve tissue samples, which demonstrated significantly increased CREBBP protein expression in BAV leaflet tissues compared with TAV leaflets (Fig. 4A and B). CREBBP mRNA expression in human leaflets was also measured, and the results demonstrated a -2.08-fold decrease of CREBBP mRNA expression in BAV leaflets compared with TAV tissues (Fig. 4C). Pearson's correlation analysis on these human tissue samples indicated that the CREBBP protein expression levels negatively correlated with miR-330-3p expression $(r=-0.897$; $\mathrm{P}<0.05$; Fig. 4D). 
A

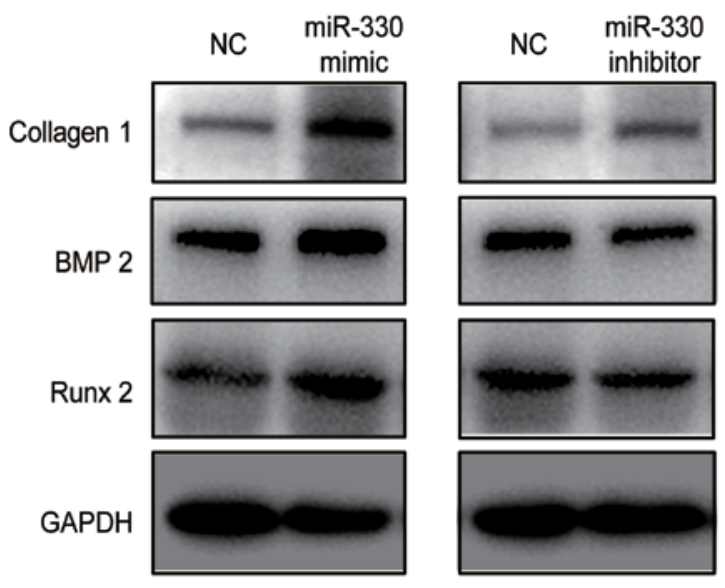

B

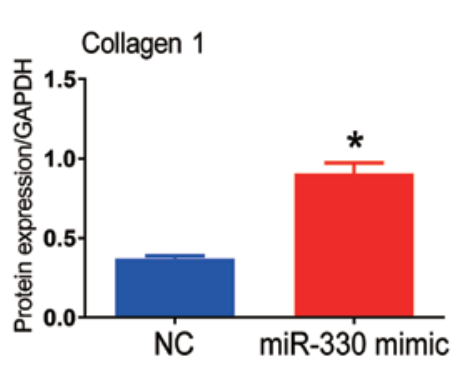

C
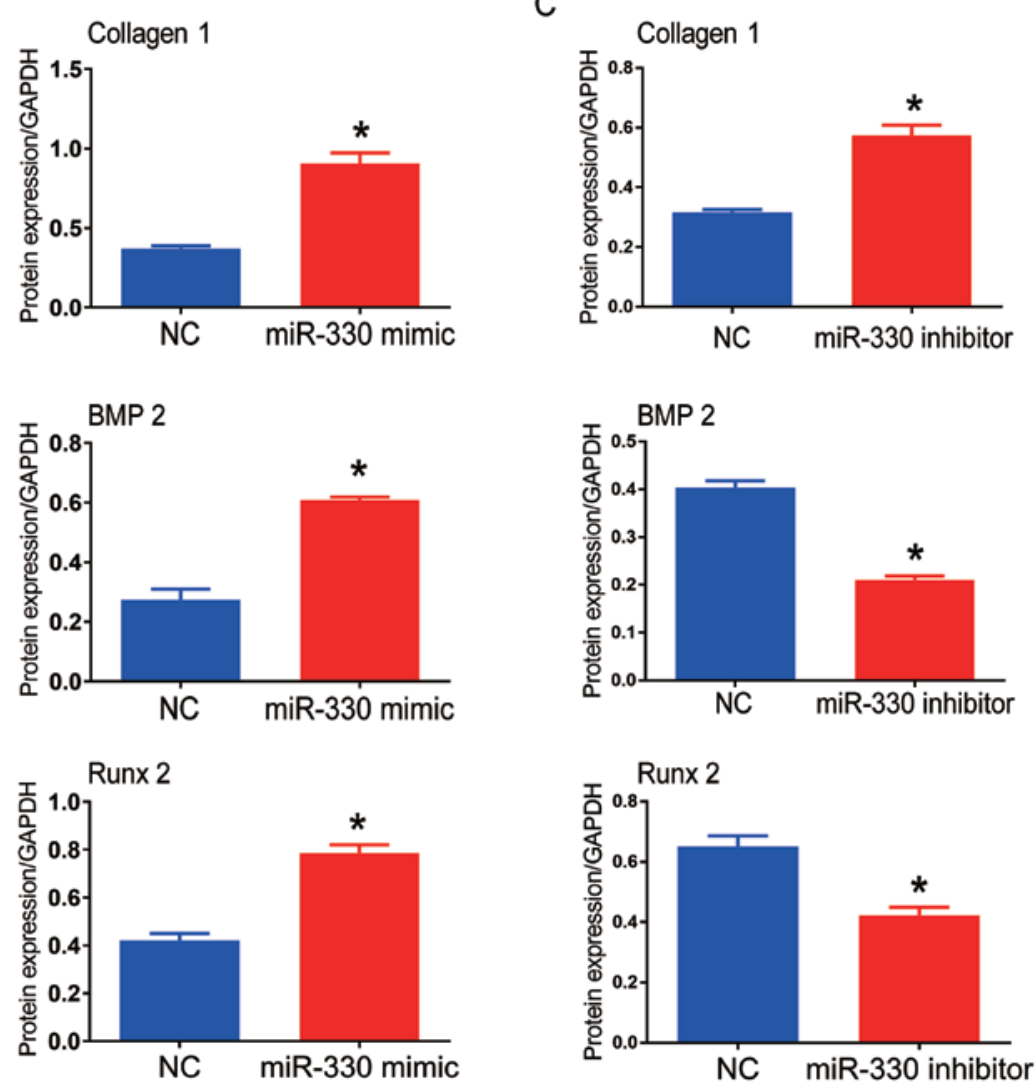

Figure 3. Expression levels of pro-calcification proteins in cultured VICs transfected with miR-330 mimic or inhibitor. (A) Western blots of pro-calcification proteins in VICs transfected with miR-330 mimic or miR-330 inhibitor. Quantification of collagen 1, BMP2, and Runx 2 protein expression levels in VICs transfected with (B) miR-330 mimic or (C) miR-330 inhibitor. Student's t-test was performed. Data are presented as the mean \pm standard deviation. ${ }^{*} \mathrm{P}<0.05$ vs. control. miR, microRNA; VICs, valvular interstitial cells; BMP2, bone morphogenetic protein 2; Runx 2, Runt-related transcription factor 2; $\mathrm{NC}$, negative control.

Computational analysis demonstrated that the seed sequence of hsa-miR-330-3p was complementary to the 56-62 nt of the 3 '-UTR in CREBBP mRNA, which is a potential binding site for miR-330-3p directly targeting CREBBP (Fig. 4E). Dual-luciferase reporter assay was subsequently performed using $293 \mathrm{~T}$ cells transfected with wild-type or point mutation vectors of the 3'-UTR region of CREBBP at the predicted binding site. Luciferase activity was significantly decreased in cells treated with miR-330-3p mimic in the wild type group compared with the negative control group, while there was no significant difference in luciferase activity between the mutated groups (Fig. 4F), indicating that miR-330-3p directly binds to the CREBBP 3'-UTR.

Furthermore, cultured VICs treated with miR-330 mimic for $36 \mathrm{~h}$ exhibited significantly lower CREBBP protein expression by -1.88 -fold compared with the control group, while treatment with miR-330-3p inhibitor significantly increased CREBBP protein expression by 1.55 -fold (Fig. 4G-I). Western blots from repeat experiments are shown in Fig. S3A and B. CREBBP mRNA expression in cultured VICs showed the same patterns to protein expression (Fig. $4 \mathrm{~J}$ and $\mathrm{K}$ ). 
A

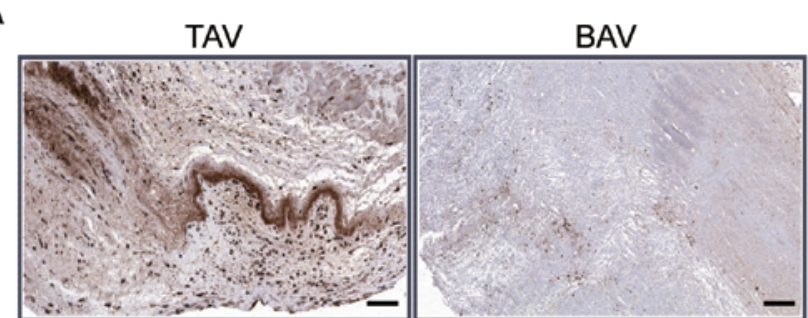

B

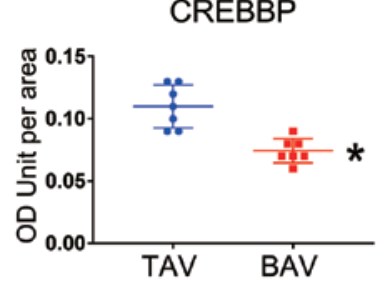

C

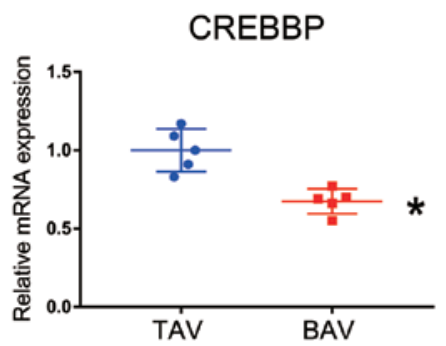

E

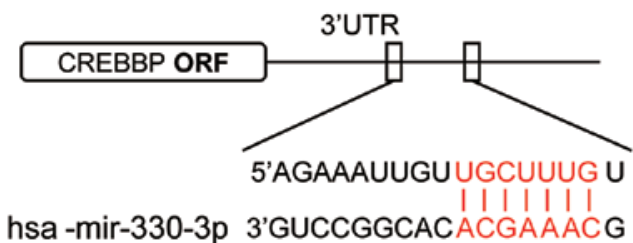

G

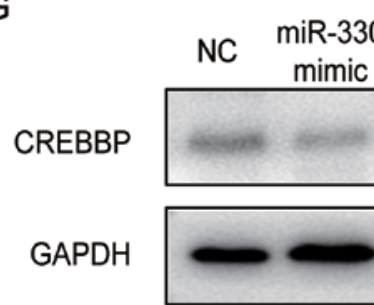

I

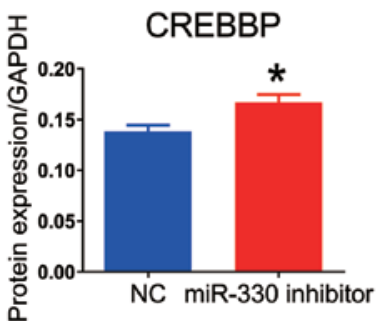

D

$J$

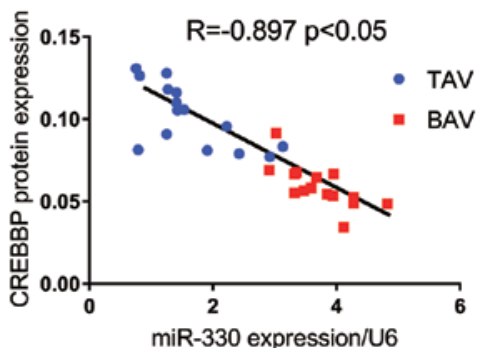

F

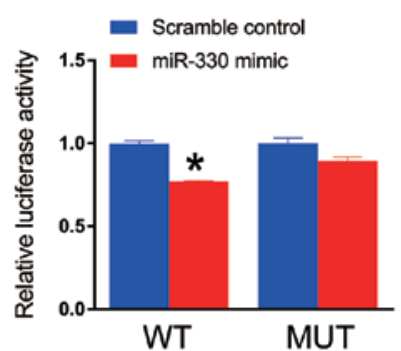

$\mathrm{H}$

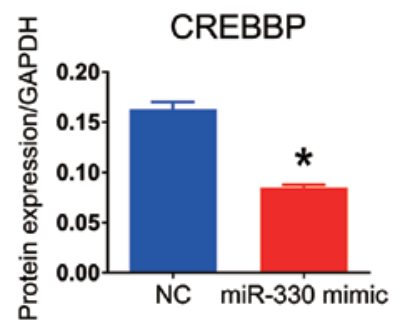

K

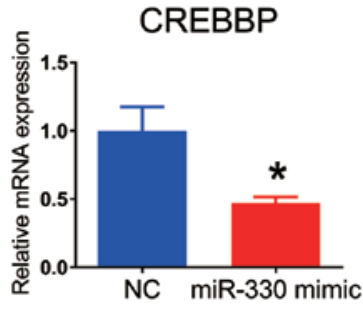

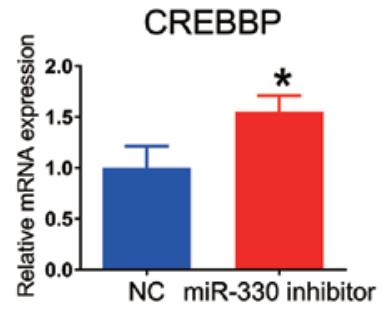

Figure 4. CREBBP is a direct target of miR-330 in both human valve tissues and in cultured VICs. (A) Representative images of human aortic valve tissue slices assessed via immunohistochemistry for CREBBP. Scale bar, $50 \mu \mathrm{m}$. CREBBP expression was lower in the human BAV group compared with the TAV group at both the (B) protein and (C) mRNA levels. " $\mathrm{P}<0.05$ vs. TAV. $n=6$ in the TAV group; $n=8$ in the BAV group. (D) Pearson's correlation analysis demonstrated that CREBBP protein levels negatively correlated with miR-330 expression. $n=15$ per group; $R=-0.897 ; P<0.05$. (E) Predicted binding sites of miR-330 with CREBBP. (F) miR-330 mimic significantly suppressed luciferase activity in 3'-UTR WT clones but not in 3'-UTR mutant clones. "P<0.05 vs. scramble control. (G) Western blots of CREBBP protein expression levels in VICs transfected with miR-330 mimic or miR-330 inhibitor. Quantification of CREBBP protein expression levels in VICs transfected with (H) miR-330 mimic or (I) miR-330 inhibitor. ${ }^{*} \mathrm{P}<0.05$ vs. TAV. CREBBP mRNA expression in VICs transfected with (J) miR-330 mimic or (K) miR-330 inhibitor. "P<0.05 vs. TAV. Student's t-test was performed. Data are presented as the mean \pm standard deviation. miR, microRNA; VICs, valvular interstitial cells; BAV, bicuspid aortic valve; TAV, tricuspid aortic valve; UTR, untranslated region; OD, optical density; ORF, open reading frame; WT, wild-type; MUT, mutant; NC, negative control; CREBBP, CREB-binding protein.

Promotion of calcification induced by miR-330-3p is suppressed by CREBBP upregulation. It was further investigated whether the calcification promotion effect of miR-330-3p depends on the downregulation of CREBBP. Since the coding sequence of CREBBP gene is too long, CREBBP protein cannot be overexpressed by the conventional construction of plasmids 
A

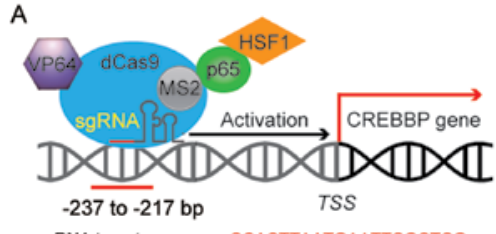

sgRNA target sequence: CCACTTAATGAATTCGCTCG

C
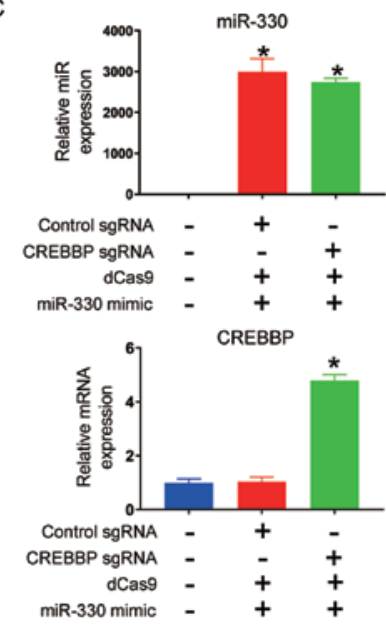

E

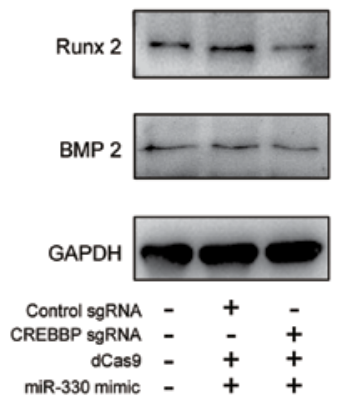

$\mathrm{F}$

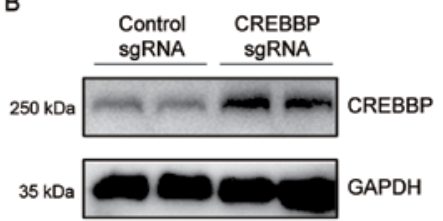

D
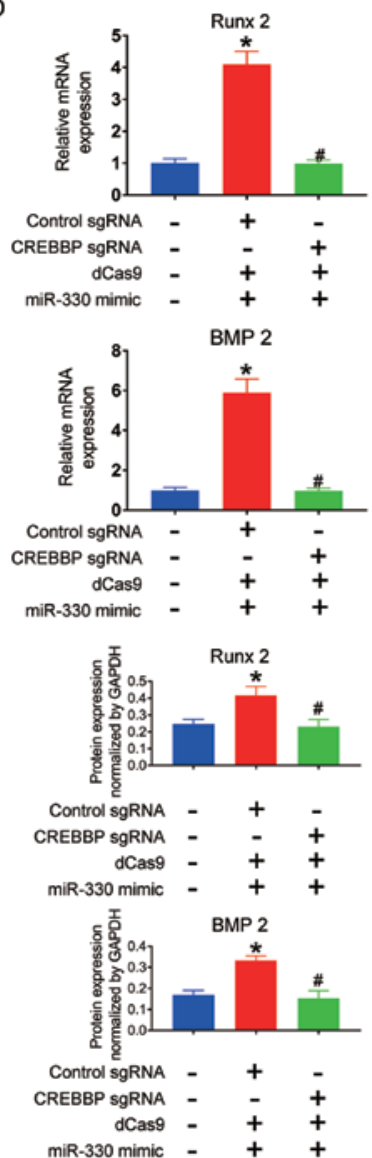

Figure 5. Promotion of calcification via miR-330 is suppressed by upregulating endogenous CREBBP in cultured VICs. (A) Schematic of CRISPRa strategy to activate CREBBP gene expression. (B) CRISPRa induction of CREBBP increased CREBBP protein expression, as shown by Western blotting. (C) VICs were transfected with either a negative control or sgRNA targeting the proximal CREBBP promoter region in the presence of miR-330 mimic. mRNA levels of miR-330 and CREBBP were determined via reverse transcription-quantitative PCR analysis. (D) mRNA expression levels of BMP2 and Runx 2 in VICs transfected with gRNA targeting CREBBP, in the presence of miR-330 mimic. (E) Western blots and (F) quantification of BMP2 and Runx 2 protein expression levels in VICs transfected with gRNA targeting CREBBP in the presence of miR-330 mimic. One-way ANOVA with Dunnett's post hoc test was performed. Data are presented as the mean \pm standard deviation. ${ }^{*} \mathrm{P}<0.05$ vs. negative control; ${ }^{*} \mathrm{P}<0.05$ vs. negative control in the presence of miR-330 mimic. miR, microRNA; VICs, valvular interstitial cells; CRISPR, clustered regularly interspaced short palindromic repeats; sgRNA, single guide RNA; BMP2, bone morphogenic protein 2; Runx 2, Runt-related transcription factor 2; CRISPRa, CRISPR activation; CREBBP, CREB-binding protein. Cas9, CRISPR-associated protein 9; HSF1, Heat shock transcription factor 1.

containing its cDNA sequence. Hence, endogenous overexpression was performed using the transcriptional activation method. CREBBP transcriptional activation was induced by CRISPR activation (CRISPRa) strategy, which exploits the targeting mechanism of an sgRNA to direct the complex to a promoter or enhancer of a gene. In the present study, CRISPRa targeted the CREBBP promoter region and significantly increased the expression of endogenous CREBBP in VICs compared with the control group (Fig. 5A and B). CREBBP mRNA expression was also significantly increased in the CREBBP sgRNA group compared with sgRNA negative control group in the presence of miR-330-3p mimic (Fig. 5C). mRNA (Fig. 5D) and protein (Fig. 5E and F; S3C and D) expression levels of Runx 2 and BMP2 significantly decreased following overexpression of CREBBP in the presence of miR-330-3p mimic, indicating that the presence of CREBBP may alleviate the degree of calcification in the high miR-330-3p environment.

BAV leaflets present more calcification-associated alterations similar to cellular alterations. To validate that upregulated microRNA-330-3p promotes calcification in patients with BAV, the positive areas of pro-calcification proteins in aortic valve samples from patients with BAV and TAV were examined. Von Kossa staining demonstrated similar calcium deposits between TAV and BAV leaflet slices (Fig. 6E). Furthermore, immunohistochemical staining of Runx 2, collagen 1, MMP 2 and MMP 9 demonstrated significantly higher positive areas in BAV samples compared with TAV samples (Fig. 6A-D). These alterations suggest a poor disorder of the interstitial function of VICs in patients with BAV compared with patients with 
A
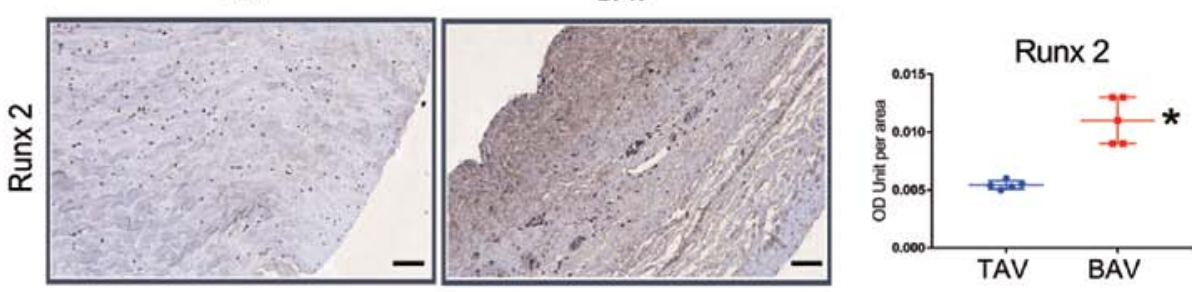

B
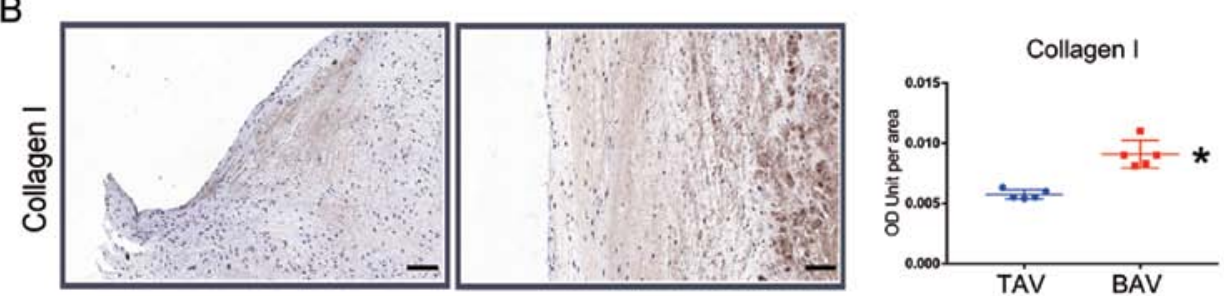

C
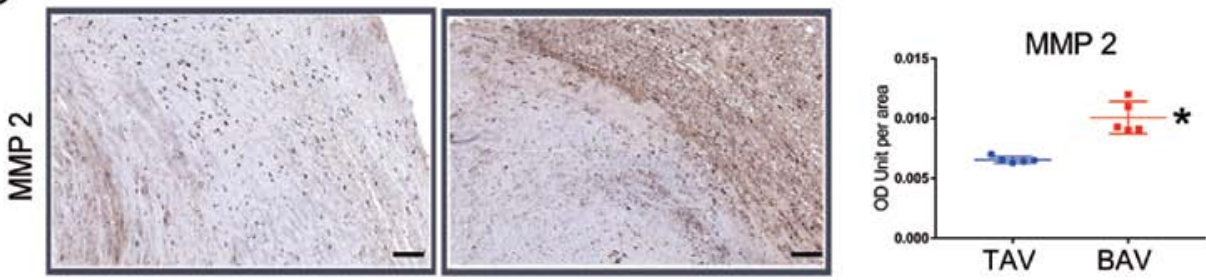

D
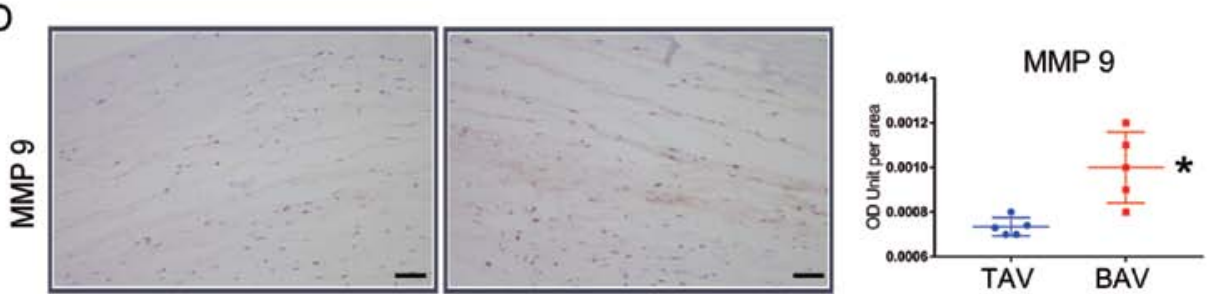

E
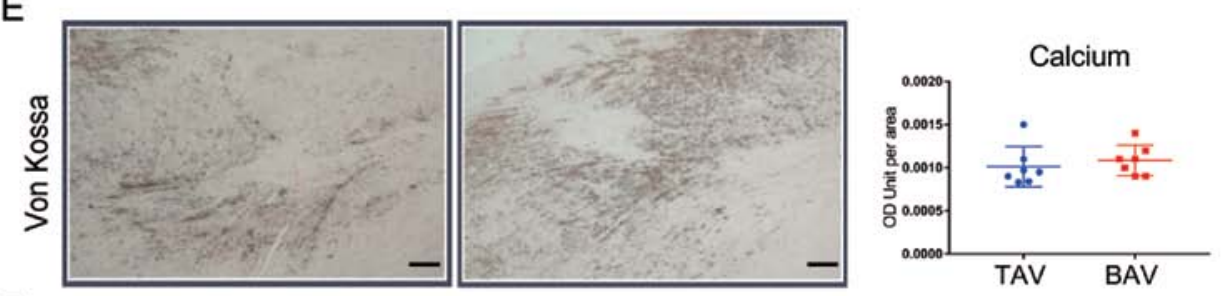

$\mathrm{F}$
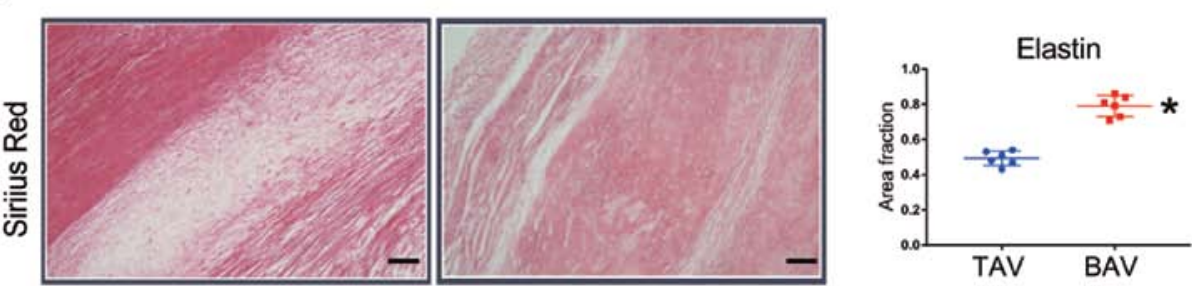

Figure 6. BAV leaflets present more calcification-associated alterations similar to cellular alteration. Representative images and quantification of immunohistochemical staining of (A) Runx 2, (B) collagen I, (C) MMP 2 and (D) MMP 9 expression in human aortic valve leaflets. (E) Representative images and quantification of Von Kossa staining for calcium deposits in human aortic valve leaflets. (F) Representative images and quantification of Sirius Red staining of elastin in human aortic valve leaflets (magnification, $\mathrm{x} 200$; scale bar, $50 \mu \mathrm{m}$ ). Student' s t-test was performed. Data are presents as means \pm standard deviation. $\mathrm{n}=6$ per group. ${ }^{*} \mathrm{P}<0.05$ vs. TAV. MMP, matrix metalloproteinase; $\mathrm{BAV}$, bicuspid aortic valve; TAV, tricuspid aortic valve; Runx 2 , Runt-related transcription factor 2 ; OD, optical density.

TAV. Furthermore, Sirius Red staining demonstrated a higher concentration of elastin in the BAV group compared with the TAV group, which is prone to calcification in a number of disease processes (Fig. 6F).

\section{Discussion}

The present study identified a total of 19 differentially expressed miRs between BAVs and TAVs via microarray analysis. Of 
the 19, only four miRs (miR-146, miR-330-3p, miR-659 and miR-663) demonstrated a significant difference between the two groups when compared with the leaflets obtained from patients with AS. The alteration in the calcification progress was indicated in sus scrofa VICs when these miRs were overexpressed or knocked down. Of the four miRs, only miR-330-3p was demonstrated to influence the calcification by remarkably accelerating its calcification progress. Thus, miR-330-3p was selected for further experimentation. Both CREBBP mRNA and protein expression levels were demonstrated to increase in BAV leaflets; however, CREBBP protein expression in human aortic valves was negatively correlated to miR-330-3p expression. Furthermore, increased miR-330-3p expression in VICs was demonstrated to decrease CREBBP mRNA and protein expression, while miR-330-3p knockdown increased CREBBP mRNA and protein expression levels. In addition, BAV leaflets presented calcification-associated alterations similar to the cellular alterations. miR-330-3p expression was upregulated in human BAV leaflets, which suggested that it may play a role in early calcification by regulating CREBBP, which, to the best of our knowledge, has not been previously reported.

$\mathrm{CBP} / \mathrm{p} 300$ interacting transactivator 1 (CiTED1) was reported to be an inhibitor for the early calcification of BAVs $(25,26)$. Lin et al $(26)$ reported that the translocation of CiTED1 from the cytoplasm to the nucleus was promoted by the parathyroid hormone in the mouse osteoblastic MC3T3-E1 subclone 14 cell line, which impaired the calcification progress. They also found that serine-to-alanine mutations at position 79 in the 63-84 domain of CiTED1 affect Runx 2 expression, which is a key protein relevant to calcification (26). CREBBP is known as a key co-activator of CiTED1 (25). Therefore, the aberrant expression of CREBBP may have impact on calcification which is consistent with the results of the present study. Furthermore, it was reported that the translocation of CiTED1 is enhanced by the protein kinase $\mathrm{C}$ activator $(27,28)$. A number of studies have set out to determine the role of miR-330-3p in different types of tumors, most of which have reported miR-330-3p-3p as a tumor suppressor gene. For example, miR-330-3p plays a critical role in glioma, as well as in breast (29), liver, esophageal (30), lung, gastric, colon and prostate cancers (31-35). Although its role in cardiovascular calcification remains unclear, Liu et al (28) has proposed that miR-330-3p directs the proliferation of several types of cells, and demonstrated its negative influence of protein kinase $C$ on the endothelial monocyte-activating polypeptide-II in the immortalized human brain endothelial cell line, hCMEC/D3. These findings indicate that miR-330-3p may be involved in the process of calcification in cardiovascular system, which was consistent with the results of the present study.

A number of classic alteration patterns are involved in calcification, including receptor translocation mediated by serine mutation and epigenetic modulation regulated by histone acetyltransferase $(19,26)$. Previous studies reported the association between CREBBP and aortic calcification, and suggested that CREBBP ultimately promotes calcification via its translocation from the cytoplasm to the nucleus (27). Taken together, the results of the present study and findings from previous studies indicated that the alteration of CREBBP via miR-330-3p might aggravate susceptibility toward early calcification observed in patients with BAV.
The functional differences between BAVs and TAVs were assessed in the leaflets of patients. MMP-2 protein, MMP-9 protein and collagen 1 expression were higher in BAV leaflets, suggesting a disorder in the matrix function. This further validated the hypothesis that upregulation of miR-330-3p in BAV leaflets inhibited CREBBP signaling and promoted fibrosis in the extracellular matrix, which ultimately causes valvular calcification.

In the present study, VICs were incubated in pro-osteogenetic medium for $48 \mathrm{~h}$ to promote cellular calcification. This medium contains a high dose of phosphate and calcium ions, which simulates an environment often observed in acute renal failure models characterized by nephric calcification (36). It was shown that the imbalance of phosphate metabolism and high blood calcium levels are the pathogenic factors in the process of vascular calcification in patients with chronic kidney disease (37). This method was successfully applied to develop calcification in valve interstitial cells in $48 \mathrm{~h}$ (19). Compared with other methods, which usually uses dexamethasone and vitamin $\mathrm{D}$ in the medium and takes $>20$ days to develop calcification in cells, the present approach was more advantageous in terms of lower incubation time and higher cytoactiveness.

However, there are limitations in the present study. The experimental setting involved a number of models, which may not be particularly relevant for BAV as it is a type of heart valve deformation at the organ level. It is difficult to find a cellular model that mimics BAV at a cellular level. However, the in vitro model of cell calcification in the present study is well established and the results are reliable in terms of the mechanisms of promoting cellular calcification. These results need further confirmation on BAV animal models, which will be investigated in the future.

The results of the present study demonstrated that microRNA-330-3p was upregulated in the stenotic aortic leaflets from patients with BAV compared with patients with TAV. Upregulation of miR-330-3p was associated with valvular calcification via CREBBP targeting, which involves remodeling of the extracellular matrix. Taken together, these results introduced a novel perspective concerning the molecular mechanism underlying accelerated valvular calcification in $\mathrm{BAV}$, which may contribute to the prevention of this disease.

\section{Acknowledgements}

Not applicable.

\section{Funding}

This work was supported by the National Natural Science Foundation of China (grant no. 81974033).

\section{Availability of data and materials}

The datasets used and/or analyzed during the current study are available from the corresponding author on reasonable request.

\section{Authors' contributions}

YS and JD conceived and designed the study. RZ, HL, JG, $\mathrm{BN}, \mathrm{HS}, \mathrm{YG}, \mathrm{CS}$ and $\mathrm{KH}$ collected the data. RZ, HL, JG, BN, $\mathrm{HS}, \mathrm{YG}, \mathrm{CS}$ and $\mathrm{KH}$ performed the experiments, acquired the 
data and prepared the diagrams. RZ, HL, JD and YS drafted the manuscript. RZ, JD and YS reviewed and edited the manuscript. All authors read and approved the final manuscript.

\section{Ethics approval and consent to participate}

This study was granted ethical approval by the First Affiliated Hospital of Nanjing Medical University Ethics Committee. Written informed consent was obtained from the patients.

\section{Patient consent for publication}

Not applicable.

\section{Competing interests}

The authors declare that they have no competing interests.

\section{References}

1. Kurabayashi M: Molecular mechanism of vascular calcification Clin Calcium 29: 157-163, 2019 (In Japanese).

2. Chen HY, Engert JC and Thanassoulis G: Risk factors for valvular calcification. Curr Opin Endocrinol Diabetes Obes 26 96-102, 2019

3. Girdauskas E and Borger MA: Bicuspid aortic valve and associated aortopathy: An update. Semin Thorac Cardiovasc Surg 25 310-316, 2013

4. Siu SC and Silversides CK: Bicuspid aortic valve disease. J Am Coll Cardiol 55: 2789-2800, 2010.

5. Cirka HA, Kural MH and Billiar KL: Mechanoregulation of aortic valvular interstitial cell life and death. J Long Term Eff Med Implants 25: 3-16, 2015

6. Gu X and Masters KS: Role of the MAPK/ERK pathway in valvular interstitial cell calcification. Am J Physiol Heart Circ Physiol 296: H1748-H1757, 2009.

7. Gu X and Masters KS: Role of the Rho pathway in regulating valvular interstitial cell phenotype and nodule formation. Am J Physiol Heart Circ Physiol 300: H448-H458, 2011.

8. Hjortnaes J, Shapero K, Goettsch C, Hutcheson JD, Keegan J, Kluin J, Mayer JE, Bischoff J and Aikawa E: Valvular interstitial cells suppress calcification of valvular endothelial cells Atherosclerosis 242: 251-260, 2015.

9. Mohler ER, Gannon F, Reynolds C, Zimmerman R, Keane MG and Kaplan FS: Bone formation and inflammation in cardiac valves. Circulation 103: 1522-1528, 2001.

10. Kaden JJ, Bickelhaupt S, Grobholz R, Vahl CF, Hagl S, Brueckmann M, Haase KK, Dempfle CE and Borggrefe M: Expression of bone sialoprotein and bone morphogenetic protein-2 in calcific aortic stenosis. J Heart Valve Dis 13: 560-566, 2004

11. Wang Y, Wu B, Farrar E, Lui W, Lu P, Zhang D, Alfieri CM, Mao K, Chu M, Yang D, et al: Notch-Tnf signalling is required for development and homeostasis of arterial valves. Eur Heart J 38: 675-686, 2017.

12. Wu M, Chen G and Li YP: TGF- $\beta$ and BMP signaling in osteoblast, skeletal development, and bone formation, homeostasis and disease. Bone Res 4: 16009, 2016.

13. Leopold JA: MicroRNAs regulate vascular medial calcification. Cells 3: 963-980, 2014.

14. Nigam V, Sievers HH, Jensen BC, Sier HA, Simpson PC Srivastava D and Mohamed SA: Altered microRNAs in bicuspid aortic valve: A comparison between stenotic and insufficient valves. J Heart Valve Dis 19: 459-465, 2010.

15. Rathan S, Ankeny CJ, Arjunon S, Ferdous Z, Kumar S, Fernandez EJ, Heath JM, Nerem RM, Yoganathan AP and Jo H Identification of side- and shear-dependent microRNAs regulating porcine aortic valve pathogenesis. Sci Rep 6: 25397, 2016

16. Patel V, Carrion K. Hollands A, Hinton A, Gallegos T, Dyo J, Sasik R, Leire E, Hardiman G, Mohamed SA, et al: The stretch responsive microRNA miR-148a-3p is a novel repressor of IKBKB, NF- $\kappa \mathrm{B}$ signaling, and inflammatory gene expression in human aortic valve cells. FASEB J 29: 1859-1868, 2015.
17. Holliday CJ, Ankeny RF, Jo H and Nerem RM: Discovery of shear- and side-specific mRNAs and miRNAs in human aortic valvular endothelial cells. Am J Physiol Heart Circ Physiol 301: H856-H867, 2011.

18. Livak KJ and Schmittgen TD: Analysis of relative gene expression data using real-time quantitative PCR and the 2(-Delta Delta C(T)) method. Methods 25: 402-408, 2001.

19. Gu J, Lu Y, Deng M, Qiu M, Tian Y, Ji Y, Zong P, Shao Y, Zheng R, Zhou B, et al: Inhibition of acetylation of histones 3 and 4 attenuates aortic valve calcification. Exp Mol Med 51: 79, 2019.

20. Meister G, Landthaler M, Dorsett $Y$ and Tuschl T: Sequence-specific inhibition of microRNA- and siRNA-induced RNA silencing. RNA 10: 544-550, 2004.

21. Nishimasu H, Shi X, Ishiguro S, Gao L, Hirano S, Okazaki S, Noda T, Abudayyeh OO, Gootenberg JS, Mori H, et al: Engineered CRISPR-Cas9 nuclease with expanded targeting space. Science 361: 1259-1262, 2018.

22. Trevino AE and Zhang F: Genome editing using Cas9 nickases. Methods Enzymol 546: 161-174, 2014

23. Gomez RA, Belyea B, Medrano S, Pentz ES and Sequeira-Lopez ML: Fate and plasticity of renin precursors in development and disease. Pediatr Nephrol 29: 721-726, 2014.

24. Devan J, Janikova A and Mraz M: New concepts in follicular lymphoma biology: From BCL2 to epigenetic regulators and non-coding RNAs. Semin Oncol 45: 291-302, 2018.

25. Yang D, Guo J, Divieti P, Shioda T and Bringhurst FR: $\mathrm{CBP} / \mathrm{p} 300$-interacting protein CITED1 modulates parathyroid hormone regulation of osteoblastic differentiation. Endocrinology 149: 1728-1735, 2008.

26. Lin Z, Feng R, Li J, Meng Y, Yuan L, Fu Z, Guo J, Bringhurst FR and Yang D: Nuclear translocation of $\mathrm{CBP} / \mathrm{p} 300$-interacting protein CITED1 induced by parathyroid hormone requires serine phosphorylation at position 79 in its 63-84 domain. Cell Signal 26: 2436-2445, 2014

27. Li SJ, Kao YH, Chung CC, Chen WY, Cheng WL and Chen YJ: Activated p300 acetyltransferase activity modulates aortic valvular calcification with osteogenic transdifferentiation and downregulation of Klotho. Int J Cardiol 232: 271-279, 2017.

28. Liu J, Liu L, Chao S, Liu Y, Liu X, Zheng J, Chen J, Gong W, Teng H, Li Z, et al: The Role of miR-330-3p/PKC- $\alpha$ signaling pathway in Low-dose endothelial-monocyte activating polypeptide-ii increasing the permeability of blood-tumor barrier. Front Cell Neurosci 11: 358,2017.

29. Wang H, Chen SH, Kong P, Zhang LY, Zhang LL, Zhang NQ and $\mathrm{Gu} \mathrm{H}$ : Increased expression of miR-330-3p: A novel independent indicator of poor prognosis in human breast cancer. Eur Rev Med Pharmacol Sci 22: 1726-1730, 2018.

30. Meng H, Wang K, Chen X, Guan X, Hu L, Xiong G, Li J and Bai Y: MicroRNA-330-3p functions as an oncogene in human esophageal cancer by targeting programmed cell death 4 . Am J Cancer Res 5: 1062-1075, 2015.

31. Min M,Peng LH, Sun G, Guo MZ, Qiu ZW and Yang YS: Aquaporin 8 expression is reduced and regulated by microRNAs in patients with ulcerative colitis. Chin Med J (Engl) 126: 1532-1537, 2013.

32. Feng L, Ma J, Ji H, Liu Y and Hu W: miR-330-5p suppresses glioblastoma cell proliferation and invasiveness through targeting ITGA5. Biosci Rep 37: BSR20170019, 2017.

33. Chen T, Yang Z, Liu C, Wang L, Yang J, Chen L and Li W: Circ_0078767 suppresses non-small-cell lung cancer by protecting RASSF1A expression via sponging miR-330-3p. Cell Prolif 52: e12548, 2019.

34. Guan A, Wang H, Li X, Xie H, Wang R, Zhu Y and Li R: MiR-330-3p inhibits gastric cancer progression through targeting MSI1. Am J Transl Res 8: 4802-4811, 2016.

35. Lee KH, Chen YL, Yeh SD, Hsiao M, Lin JT, Goan YG and Lu PJ: MicroRNA-330 acts as tumor suppressor and induces apoptosis of prostate cancer cells through E2F1-mediated suppression of Akt phosphorylation. Oncogene 28: 3360-3370, 2009.

36. Pirklbauer M and Mayer G: The exchangeable calcium pool: Physiology and pathophysiology in chronic kidney disease. Nephrol Dial Transplant 26: 2438-2444, 2011.

37. Goodman WG, Goldin J, Kuizon BD, Yoon C, Gales B, Sider D, Wang Y, Chung J, Emerick A, Greaser L, et al: Coronary-artery calcification in young adults with end-stage renal disease who are undergoing dialysis. N Engl J Med 342: 1478-1483, 2000.

This work is licensed under a Creative Commons Attribution-NonCommercial-NoDerivatives 4.0 International (CC BY-NC-ND 4.0) License. 